Culture et histoire dans l'espace roman

\title{
La gloria de los niños de Luis Mateo Díez : Pulgar ou le parcours initiatique d'un Petit Poucet dans les ruines de la guerre
}

\section{Gregoria Palomar}

\section{(2) OpenEdition}

Journals

Édition électronique

URL : https://journals.openedition.org/cher/11709

DOI : $10.4000 /$ cher. 11709

ISSN : 2803-5992

Éditeur

Presses universitaires de Strasbourg

\section{Édition imprimée}

Date de publication : 1 décembre 2012

Pagination : 203-214

ISBN : 978-2-35410-049-0

ISSN : 1968-035X

\section{Référence électronique}

Gregoria Palomar, «La gloria de los niños de Luis Mateo Díez: Pulgar ou le parcours initiatique d'un Petit Poucet dans les ruines de la guerre », reCHERches [En ligne], 9 | 2012, mis en ligne le 14 février 2022, consulté le 17 février 2022. URL : http://journals.openedition.org/cher/11709 ; DOI : https:// doi.org/10.4000/cher.11709

\section{cc) (†)}

Ce(tte) œuvre est mise à disposition selon les termes de la Licence Creative Commons Attribution -

Pas d'Utilisation Commerciale - Partage dans les Mêmes Conditions 4.0 International. 


\title{
La gloria de los niños de Luis Mateo Díez: Pulgar ou le parcours initiatique d'un Petit Poucet dans les ruines de la guerre
}

\author{
Gregoria Palomar \\ Université Paul Verlaine - Metz
}

Selon Tzvetan Todorov, toute œuvre littéraire est «incluse dans un par les œuvres déjà existantes et c'est là qu'elle s'intègre» (Todorov: 132). On peut considérer qu'un des éléments qui ont fondé ce socle littéraire est l'ensemble des contes populaires, présents dans toutes les cultures.

Marie-Louise von Franz, dans son étude intitulée L'interprétation des contes de fées (von Franz 2003), rappelle que les récits des contes étaient la principale forme de distraction dans les populations agricoles. C'est l'expérience qu'a vécue Luis Mateo Díez, qui a souligné, à plusieurs reprises, l'importance des calechos (avant le dîner) et filandones (après le dîner), auxquels il assistait pendant son enfance dans les montagnes de León, alors que le village était isolé par la neige. Sa vocation littéraire est certainement née de ces expériences de l'oralité, et de l'imprégnation progressive de tout un substrat folklorique qu'il a fait sien.

Cet intérêt pour les contes apparaît également dans sa préface à un recueil de Miguel Díez et Paz Díez Taboada intitulé La memoria de los cuentos, un viaje por los cuentos populares del mundo. Pour Luis Mateo Díez, la littérature est issue de ces contes populaires, présents dans notre mémoire collective et indispensables à toute société

1 «La perpetuidad de los cuentos está garantizada en los libros que ahora contienen su memoria, también en las infinitas memorias que hacen posible su pervivencia, mantenida 
Cependant, si la référence aux filandones était très présente dans ses premières œuvres, très marquées par l'expression de l'oralité, en particulier dans son recueil Relato de Babia, publié en 1991, Luis Mateo Díez semblait avoir délaissé cet univers pour créer progressivement un territoire littéraire, simplement dénommé "El Territorio", au centre duquel se trouve la contrée de Celama. Il s'agit d'un espace imaginaire délimité, voire emprisonné, par deux rivières, l'Urgo et l'Esla, une région dont les habitants, résignés à la misère, n'ont pas, comme à Babia, la magie de l'oral et la convivialité pour s'évader du quotidien, les légendes semblant inexistantes à Celama.

La gloria de los niños, roman publié en 2007, se situe aux confins de ce territoire imaginaire, en bordure de la rivière Urgo, mais ce récit semble rompre avec les précédents car, si le monde de l'enfance et de l'adolescence est présent dans l'univers romanesque de Luis Mateo Díez, pour la première fois le protagoniste est un enfant prénommé Pulgar, montrant ainsi la volonté, de la part de l'auteur, de se référer à l'univers des contes populaires. Pulgar est un enfant qui, comme le Petit Poucet du conte de Perrault, est soumis à une épreuve initiatique, celle de retrouver ses frères et sœurs, dans un cadre hostile, celui d'un immédiat après-guerre. Dans un univers parfois onirique, souvent labyrinthique, il devra effectuer plusieurs tâches proposées par des adultes rencontrés au gré de ses pérégrinations, avant de mener à bien sa mission et de partir seul vers l'âge adulte. Malgré l'allusion directe au Petit Poucet, l'univers proposé est bien celui de Luis Mateo Díez, dans ce territoire qui sert de cadre symbolique aux errances des hommes, mais nous verrons dans quelle mesure les emprunts à des schémas préexistants et les références à l'univers des contes populaires aident à l'interprétation de ce texte et donnent une autre dimension à ce récit du vingt-et unième siècle.

Le cheminement du héros, Pulgar, nous plonge dans un univers qui rappelle les quêtes de certains héros de contes populaires. Le récit se situe dans l'immédiat après-guerre dans une ville de Borenes détruite par les bombardements. Ses rues presque toujours embrumées et ses habitants solitaires, souvent à la limite de la folie, portent les stigmates d'un conflit toujours évoqué comme "la Contienda $»^{2}$. Dans cet environnement dévasté

sobre el convencimiento de que los cuentos son necesarios, y es su necesidad la que hace que, como dice Roland Barthes, estén presentes en todos los tiempos, en todos los lugares, en todas las sociedades» (Miguel Díez R., Paz Díez-Taboada 1998:16).

2 «Ese Barrio extremo menos devastado que ninguno y donde los avatares de la ciudad sitiada fortalecieron cierto intermitente refugio, hasta que la Contienda finalizó » (Díez 2007: 24) «La costurera recobró las habilidades y todavía durante unos años, en el tiempo desolado de la Contienda, acumuló la labor y los compromisos [...]» (Díez 2007: 127). 
Pulgar, un enfant d'une douzaine d'années (il a fait sa communion quatre années auparavant ${ }^{3}$ ), aîné d'une fratrie qui se compose également de Ninfa et de deux jumeaux, Vero et Nino, et dont la mère est morte pendant la guerre, se voit confier par son père moribond une mission: retrouver ses frères et sœur disparus quand l'immeuble où ils vivaient a été évacué.

Pulgar, après bien des épreuves et des errances dans une labyrinthique ville en ruines, retrouve d'abord l'un de ses frères jumeaux, Nino, recueilli par une famille aisée, mais dont le mutisme trahit l'inadaptation à cette nouvelle vie. Puis il retrouve sa sœur Ninfa, que des prostituées ont recueillie et qui se fait maintenant appeler Princesa. Enfin, il retrouve dans un bidonville et dans une extrême misère physique et psychologique, Vero, qu'il laisse sur le palier de la maison où vit Nino, afin que les deux jumeaux, incapables de supporter la séparation, puissent grandir ensemble. Quant à sa sœur Ninfa, il doit se résigner à la confier à un orphelinat de religieuses. Pulgar a ainsi le sentiment d'avoir accompli la mission que lui avait assignée son père, mais il part seul et nous saurons plus tard qu'il a grandi loin de la chaleur familiale.

Nous sommes donc en présence d'un protagoniste que Luis Mateo Díez a voulu, par le nom, rapprocher des personnages des contes populaires et dès la première lecture les renvois au héros du conte de Perrault sont manifestes. Nous savons peu de chose du protagoniste et son nom semble lui suffire pour s'identifier puisqu'il se présente toujours de la même façon, concise: "Soy Pulgar», nom qui, comme la plupart des héros des contes de fées, l'individualise. Le dernier chapitre, centré sur l'adulte qu'est devenu le protagoniste, nous apprendra qu'il ne s'agit là que d'un surnom qui disparaît avec l'enfance ou peut-être limité au temps de la quête de ses frères et sœur.

Le Pulgarcito du conte de Perrault n'est plus, dans le récit de Luis Mateo Díez, le plus jeune de la fratrie mais l'aîné, et perd son diminutif pour Pulgar, mais tous les personnages qu'il croise soulignent sa petitesse et sa fragilitét. C'est donc à la fois un adolescent qui prend en charge sa famille et un enfant aussi chétif que Pulgarcito qui doit affronter le monde des adultes en raison

3 «La primera comunión la hice hace cuatro años» (Díez 2007: 173).

4 «No me engañes, el mayor nunca puede ser el más pequeño, apenas te distingo. Eres un niño de muy poca enjundia. No se te aprecia» (Díez 2007: 32). La Marraine se souvient qu'il était tout petit en venant au monde: «Eras así de guapo, más diminuto, casi invisible, daba miedo sujetarte porque te podías escurrir. Una miniatura de la que no podía pensarse que creciera [...]» (Díez $2007: 128$ ). 
de l'absence des parents protecteurs. Mais c'est aussi, comme le héros du conte de Perrault, un enfant vif et attentif aux autres, ainsi que le remarque la marraine: "Eres de esos niños sagaces [...] que tienen la viveza de los niños más listos» (Díez 2007: 13).

C'est un enfant abandonné comme le Petit Poucet, mais alors que les parents de celui-ci doivent se débarrasser de leurs enfants car ils ne peuvent plus les nourrir le père de Pulgar, incapable d'assumer ses responsabilités vis-à-vis de ses enfants, les a laissés livrés à eux-mêmes, affrontant seuls la mort de leur mère et les dangers de la ville en guerre. Les deux héros sont conscients des faiblesses de leurs parents: le Petit Poucet écoute la nuit les conversations de ses parents, Pulgar observe sa mère de plus en plus fatiguée et prend en charge ses frères et sœur: "Desde muy pronto supo Pulgar que existían obligaciones familiares que le correspondían a él» (Díez 2007: 46).

Quand l'adulte se souvient de sa vie d'enfant, nous voyons que c'est lui qui, déjà du vivant de sa mère puis après son décès, s'occupe de trouver à manger dans une ville en ruines, suppléant ainsi à l'absence du père ${ }^{5}$. Manger devient alors le leitmotiv du récit, comme il l'est pour le Petit Poucet (Saupé 1997 : 219), Pulgar devant compter sur le chapardage ou la générosité des adultes pour apaiser sa faim.

Cependant, ce n'est pas la faim qui justifie ses déplacements mais la quête, la recherche de ses frères et sœur qui le mène dans les divers endroits que les adultes lui indiquent. Il parcourt ainsi tous les quartiers de la ville jusqu'aux confins de celle-ci. Nous nous trouvons ainsi face au thème récurrent de la route, avec de constants déplacements du héros. L'aventure du Petit Poucet l'oblige à des périples incessants, le héros accomplissant huit trajets, exprimés dans trente-deux verbes de mouvement (Saupé 1997: 220-221), Pulgar, pour sa part, quitte Larmina, le quartier de son enfance et se rend d'abord à la Citeria (Díez 2007: 24), un quartier de Borenes, puis, il suit Rovira jusqu'à la Ciudadela (Díez 2007: 68), puis encore à Larmina, rue Cedero, sur les indications de Mundo (Díez 2007: 81), puis il doit s'aventurer jusqu'au «Poblado de Colma» à la recherche de l'un des jumeaux (Díez 2007: 209).

Ces itinéraires nous font découvrir le paysage dévasté et sordide de Borenes après la «Contienda»; cependant, Pulgar, enfant de la ville, est

5 «Era Pulgar quien regresaba, unas veces con el hatillo más colmado y otras sin que la rebusca hubiese dado resultado alguno, aunque en tales ocasiones se arriesgaba a distraer una fruta en algún puesto del Mercado, para lo que no había adquirido la mínima habilidad y en cuya acción padecía el mayor nerviosismo" (Díez 2007: 28). 
caractérisé par sa Marraine comme "un niño del bosque», une forêt qui, dans cette cité du Territorio, ne peut qu'être symbolique. Nous remarquons d'ailleurs que si dans les contes traditionnels, les vieilles fées vivent souvent "au fin fond de forêts épaisses» (Belmont 1999: 17), Pulgar doit affronter d'inquiétantes vieilles femmes au fin fond des sombres immeubles, comme doña Comba ou une vieille femme menaçante qui le repousse et le renvoie brutalement (Díez 2007: 170). Les rues et les immeubles vétustes, lieux de rencontres inquiétantes, renvoient donc aux forêts des contes que le héros doit traverser pour réaliser la tâche qui lui est proposée ou pour choisir, comme le petit Chaperon Rouge, entre deux chemins possibles et prendre ainsi en charge son destin.

Ce long parcours semé d'embûches et de choix difficiles pour l'enfant est par ailleurs symbolisé, dans le récit, par une image récurrente de couloirs interminables, comme par exemple les innombrables couloirs que l'enfant doit parcourir avant d'arriver à la chambre de son père «Pulgar llegó al pie de la cama de su padre, después de infinitas vueltas y revueltas, cuando ya el cansancio le había hecho esconderse en el hueco de las escaleras [...]» (Díez 2007: 10). Le jeune garçon ressent cette même sensation d'égarement et de décision difficile à prendre quand il s'aventure dans la maison de doña Comba: «el vacío dificultó la decisión de Pulgar para acercarse a la puerta de la última habitación» (Díez 2007: 31).

À l'hôpital où est son père, Pulgar contemple la longue double rangée de lits de la chambre qu'il doit traverser pour trouver un verre d'eau pour son père: «La doble fila resultaba interminable» (Díez 2007: 14). L’image du long trajet à parcourir, parsemé d'intersections à franchir, devient la représentation symbolique d'une épreuve initiatique que le père impose à son fils, un long cheminement qui le pousse hors de l'enfance. Comme le souligne Propp dans sa Morphologie du conte, ce parcours débute par un méfait, en l'occurrence la destruction provoquée par le conflit, méfait collectif et l'abandon du père, méfait individuel. Vient ensuite la proposition de la tâche qui naît de la constatation d'un manque (Propp 1970: 45); ce sont ici des êtres humains qui manquent, les frères et sœur dispersés, justifiant l'envoi du héros à leur recherche mais un autre manque apparaît, plus difficile à résoudre, celui de la présence réconfortante des parents, d'une maison, d'un foyer.

Dans l'accomplissement de la tâche, le héros peut recevoir l'aide d'un adjuvant (Propp 1970: 55). Si le Petit Poucet est un héros qui n'a besoin d'aucun adjuvant pour accomplir sa mission, Pulgar est aidé par plusieurs 
personnes et aussi par un chien sans nom, le chien, toujours présent aux moments les plus difficiles. C'est finalement Mundo qui lui indique où trouver son petit frère Nino, et c'est dans le bidonville simplement appelé «El Barrio " que Pulgar termine l'accomplissement de la tâche. Nous voyons donc que les personnages et les lieux déterminants ont des noms génériques et descriptifs qui nous renvoient à l'univers symbolique des contes: Mundo est celui qui fait comprendre le monde au jeune garçon, il apparaît plusieurs fois dans le récit, contrairement aux nombreux figurants qui n'apparaissent qu'une fois. Un autre personnage est omniprésent dans le souvenir du narrateur, et porte un nom symbolique: la Madrina, la Marraine, soutien moral indispensable pour le jeune Pulgar. Ninfa elle-même est, comme le laisse attendre son nom, caractérisée par sa grâce insouciante et prendra comme nom Princesa, symbole également de beauté dans le monde des contes où elle s'est réfugiée. Cette présence de personnages au nom symbolique renvoie à une typologie des personnages basée «non sur la psychologie mais sur l'unité des actions que le récit leur impartit» (Barthes 1977: 33) faisant de ces personnages des archétypes plus que des héros personnalisés des romans modernes.

Pour l'aider à mener à bien sa quête, les adultes soumettront Pulgar à plusieurs épreuves qualifiantes: le jeune garçon doit aider Rovira à voler de la nourriture (Díez 2007: 59), rappelant ainsi la formation d'un jeune pícaro, puis ce même Rovira lui enjoint de lui trouver des vêtements (Díez 2007: 146) et un billet de train pour qu'il puisse s'enfuir (Díez 2007: 151). Rita, qui lui a permis de retrouver sa sœur, lui demande d'aller voir qui lui transmet des mots d'amour dans les pains qu'elle achète. Pulgar se rend alors à la boulangerie et trois boulangers, les trois frères, lui demandent de choisir lequel des trois pétrit le mieux le pain, et méritera ainsi l'amour de Rita (Díez 2007 : 205).

Ces trois boulangers nous rappellent un autre élément essentiel dans les contes de fées: la permanence du chiffre trois. La fratrie dont fait partie Pulgar est en quelque sorte composée de trois éléments: Pulgar, Ninfa, et les deux jumeaux indissociables, mais la séparation des jumeaux rompt cet équilibre. Ceux-ci ne peuvent d'ailleurs pas vivre l'un sans l'autre: une fois séparés, tous deux sont muets, comme frappés d'autisme et Pulgar préférera partir seul pour leur permettre de grandir ensemble. Ainsi, ce sont trois tâches que doit accomplir Pulgar: en premier lieu, faire sortir Ninfa de la maison de prostituées où elle ne pouvait grandir dans des conditions satisfaisantes, retrouver Vero et s'assurer qu'il est heureux, sortir Nino du 
bidonville où il survit démuni de tout. Cette série de trois tâches permettra à Pulgar de rétablir l'équilibre initial.

La gloria de los niños emprunte donc bien des éléments constitutifs au conte, mais nous constatons que celui-ci est présent sous une autre forme: les personnages ont eux aussi recours au conte pour affronter la réalité, dans une sorte de jeu d'enchâssement, de conte à l'intérieur du conte. Les enfants, en effet, connaissent l'univers des contes qu'ils ont entendus avant la guerre et la séparation. Ninfa se souvient que sa mère leur racontait l'histoire de la petite fille qui s'était perdue dans la forêt, ou encore que les prostituées la comparaient à la princesse Petit Pois (Díez 2007: 192). La petite fille a recours à ces contes pour évoquer sa propre histoire:

había una niña que no tenía madre y un día, cuando estaba en casa con sus hermanos, dijo la gente que tenían que irse porque la casa se iba a caer. [...] Entonces esa niña se quedó sola. ¿Te acuerdas de aquella otra, en el cuento que contaba mamá, que se perdió en el Bosque?... Pues lo mismo. (Díez 2007: 191)

Quand ces enfants perdus se trouvent confrontés à une réalité insoutenable, ils se réfugient dans l'univers des contes, dans ces constructions imaginaires indispensables à leur survie, puisque l'on considère que «Plus les conditions extérieures sont défavorables au développement de l'individu et plus il aura tendance à sécréter une protection symbolique de cet ordre» (Von Franz 1974: 96). Le conte serait alors pour l'enfant une façon d'exprimer l'inexprimable, de concrétiser ses angoisses. Ainsi, Ninfa, qui a été brutalement arrachée à son environnement familial, qui a vécu la douleur de la séparation, qui a été accueillie dans une maison close, dont on peut supposer qu'elle ne peut regarder en face cette réalité, s'évade dans un univers féerique. La jeune femme qui donne à Pulgar des nouvelles de sa sœur lui explique "Ahora prefiere que la llamen Princesa» (Díez 2007: 185). Quand Ninfa parle d'elle-même, l'utilisation systématique de la troisième personne, établit une distance avec cette enfant orpheline qu'elle ne veut pas être.

Ninfa, quand elle parle d'elle à la troisième personne, a donc choisi de se couper de son univers précédent, insupportable puisqu'associé à la mort de sa mère et à l'abandon du père. Fuyant la réalité, elle décide de se donner une nouvelle identité, Princesa, qui lui permet de se réfugier dans un monde merveilleux, où les prostituées deviennent des «hadas madrinas" (Díez 2007: 192). Propre et parfumée, elle semble avoir oublié ses deux frères et ne pas tenir à retrouver la maison familiale quand elle demande à son frère qu'elle vient de rejoindre: «¿Vamos a volver a casa o buscamos 
el Palacio de las Hadas o nos hacemos artistas del circo Malabares?» (Díez 2007: 187-188).

Mais elle est capable de raconter à son frère qu'elle a été une enfant abandonnée recueillie dans une maison où il y avait beaucoup de femmes, introduisant sa narration par une remarque très lucide: "Lo cuento como se lo contaba a mamá, no como se lo contaría a los gemelos. Los gemelos no lo entenderían" (Díez 2007: 190). Il y a donc une volonté de la part de cette enfant de se réfugier dans un univers idyllique grâce au seul moyen dont elle dispose, l'imagination. Le passage à la première personne indique ces moments de lucidité. Elle doit d'ailleurs revenir à la réalité quand Pulgar la laisse chez les religieuses, la première personne étant le signe de son incapacité à se réfugier dans un autre monde face à une réalité qui s'impose douloureusement: «No sé si no me gustaba más la casa de las hadas» (Díez 2007: 198). On peut supposer que Ninfa va maintenant abandonner le monde de l'enfance.

C'est aussi la force de l'imagination comme refuge qu'essaie de proposer Pulgar à son frère Vero quand il le retrouve, un enfant sale, en haillons, replié sur lui-même, indifférent à ce qui l'entoure et à son frère aîné. Le conte de Verines et Nines, deux frères que l'on ne pouvait distinguer, permet au frère aîné d'évoquer l'amour de la mère, du grand frère et de cet enfant que le vieux roi reconnaît malgré ses haillons: «El cuento que más le gustaba a Ninfa de todos los que contaba la Madrina era aquel en el que el Rey, viejo y ciego, reconocía al hijo que venía disfrazado como un vasallo después de muchos años. Besaba la mano al Rey» (Díez 2007: 217). Le conte permet de ramener timidement Vero à la réalité, il ne baise pas la main de son frère, comme l'avait fait le Roi du conte, mais le léger frôlement qu'obtient Pulgar est une immense victoire.

L'enfant semble donc trouver une réponse à un traumatisme qu'il ne peut comprendre dans le conte qu'invente pour lui son frère. C'est grâce au conte qu'il peut découvrir la signification de sa souffrance et espérer en sortir grandi car, comme le souligne Bruno Bettelheim, le conte permet de faire comprendre à l'enfant que ces difficultés font partie de l'existence humaine, mais qu'en les affrontant on finit par surmonter les épreuves (Bettelheim 1976: 20).

Luis Mateo Díez se sert donc du conte pour représenter le traumatisme de ces enfants, qui vivent, sans la comprendre, la déchirure de la guerre. Pulgar, en tant qu'aîné, doit assumer trop tôt les responsabilités qui ne sont pas celles d'un enfant. Le parcours dont l'adulte se souvient est une 
succession d'étapes plus difficiles les unes que les autres qui le mènent vers des lieux où les enfants n'ont pas leur place, tels que l'hôpital où son père agonise, la taverne, la maison close. L'enfant prend conscience de la souffrance des adultes car ceux-ci lui racontent leurs épreuves, leurs doutes, sans se soucier de son jeune âge et peu à peu Pulgar découvre la complexité de l'univers qui l'entoure, chaque adulte rencontré lui donnant sa propre vision du monde. La discordance, par exemple, entre le discours de Rita, qui accuse Enero de la délaisser et la version d'Enero, qui pense que Rita est une malade qui le poursuit, lui montre qu'il n'existe pas de vérité unique (Díez 2007: 109-110).

Le retour à l'innocence de l'enfance est impossible dans ce monde en guerre, peuplé d'adultes tourmentés, mais Pulgar, malgré tout, veut s'accrocher à l'univers de l'enfance. Et c'est aussi l'univers des contes qui pourrait lui servir d'échappatoire. Celui-ci est symbolisé par la présence constante de la Marraine, personnage traditionnellement protecteur, qui, par son discours, semble être la garante de l'innocence des enfants, «esos niños buenos y listos que en los cuentos demuestran su bondad y su inteligencia» (Díez 2007: 55). Dans un monde hostile, face à une guerre qui implique aussi les enfants, elle marque sa volonté de les préserver: «No hace falta que los niños se hagan hombres antes de tiempo" (Díez 2007: 55). La Marraine l'aide à comprendre le monde en lui laissant entendre que "el mundo está lleno de pistas» (Díez 2007: 212), des chemins qu'il doit parcourir et choisir lui-même pour grandir. Pulgar, en se souvenant sans cesse du discours de la Marraine, essaie de s'accrocher à l'enfance, à l'insouciance, mais la Marraine, comme la mère, a disparu, elle n'est plus là pour préserver l'innocence de Pulgar. Elle restera cependant un réconfort moral, celle qui lui a toujours donné une direction et son image radieuse préservée est un lien impérissable avec l'innocence première du nouveau-né .

L'enfant doit affronter la perte de cet adjuvant protecteur mais aussi, et surtout reconnaître la mort de sa mère, étape essentielle dans le passage au monde adulte. Il a vu sa mère morte, victime d'une balle perdue, un foulard violet sur la tête, une vision qui est restée gravée dans son subconscient, mais que seul l'adulte est capable d'affronter7. La réaction de l'enfant est le déni, le

6 «como si todo lo que a ella concernía tuviese que ver con otro recuerdo primitivo en el que Pulgar era un recién nacido que estaba en sus brazos» (Díez 2007: 128).

7 «Fue el tiempo, y con él la edad, quien restituyó el recuerdo del cuerpo tendido en medio de la calle, lo que Pulgar vislumbró en la mañana en que regresaba a casa, [...]» (Díez 2007: 18). 
refus de conserver l'image du cadavre de sa mère, en maintenant la présence persistante dans les rêves de la mère qui veille sur le repos de son fils. Quitter l'enfance, c'est accepter l'image de la mère morte, prendre conscience de la réalité de la perte, c'est aussi assumer les responsabilités qui viennent en grandissant. Pulgar, cet enfant à qui l'on donne trop vite des responsabilités d'adulte, franchit cette étape quand son père le charge de retrouver ses frères et sœurs, initiant ainsi le périple initiatique qui le fera abandonner complètement l'enfance. Ce nouveau départ lui donne aussitôt la conscience de sa condition d'orphelin: "Entonces recordó el cuerpo tendido de su madre en medio de la calle, la gente agolpada, los gritos de amenaza y súplica entre los que corrían, mientras todavía se oyeron disparos, y decidió marcharse» (Díez 2007: 16).

Il devient donc adulte en acceptant la mort de sa mère et en abandonnant une vision schématique du monde, confronté à un dilemme dramatique à l'instant où il perçoit que le bonheur du petit frère n'est pas forcément le sien. Les jumeaux ne peuvent pas vivre séparés, leur mutisme, signe d'une terrible souffrance intérieure, est l'expression du traumatisme qu'engendre la guerre et la séparation des familles. Mais Pulgar vit ce même écartèlement en décidant de laisser ses frères et sœur là où ils pourront grandir dans les meilleures conditions. Pour le bonheur de ses frères et sœur, c'est lui qui se retrouve seul avec «la conciencia de la absoluta orfandad en que quedaba, ahora que todo estaba resuelto» (Díez 2007 : 219).

C'est ce choix terrible qui le fait sortir définitivement du monde de l'enfance et atteindre l'état final, celui de la glorification, cinquième étape définie par Paul Larivaille dans son article "L'analyse (morpho)logique du récit» (Larivaille 1974: 376). Nous pourrions dire, comme l'affirme Lilyane Mourey, dans son Introduction aux contes de Grimm et de Perrault, à propos du Petit Poucet que Pulgar «vit une réelle initiation aux dangers et aux impératifs de la vie» (Mourey 1970: 50), abandonnant ainsi ce que Luis Mateo Díez appelle «el mundo cerrado de la infancia» et que l'adulte essaie de retrouver en vain comme l'exprime la phrase qui conclut le récit: «- Vienes conmigo... - dijo entonces el hombre [l'adulte qu'est devenu Pulgar], como si las palabras refrendaran una orden o una súplica, pero el niño se había dado la vuelta y se alejaba por los escombros con las manos en los bolsillos.» (Díez 2007: 224). Si, selon Pierre Larivaille, «La tradition du genre conte exige la présence d'un héros qui, à terme, l'emporte» (Larivaille 1974: 375), le héros de Luis Mateo Díez fait partie de ces perdants si chers au romancier, qui luttent malgré tout pour survivre sans vraiment triompher. 
Pulgar participe à l'élaboration de cet univers que l'on retrouve dans de nombreuses fictions de Luis Mateo Díez, habité par des hommes en quête constante, qui ne veulent pas s'avouer vaincus face à une société qui est souvent représentée comme défaitiste ou corrompue. Dans La gloria de los niños, le choix du titre traduit une volonté de généralisation (alors que les titres des contes font souvent référence à un héros individualisé, tel que $L e$ petit Poucet, Le Chat Botté, La Petite Sirène), qui fait de Pulgar le symbole de la déchirure provoquée par la guerre, non seulement la guerre civile espagnole, mais toute guerre, comme le traduit le terme de Contienda.

Une première approche de La gloria de los niños nous montre donc que le récit correspond au schéma défini par Marie-Louise von Franz: les contes de fées commencent par une situation horrible puis «Le héros est le restaurateur d'une situation consciente juste. Il est le seul moi capable de rétablir une situation saine et normale là où tous les moi de cette tribu ou nation dévient de leur modèle instinctif fondamental de totalité.» (Von Franz 2003: 82).

Luis Mateo Díez, en empruntant à l'univers du conte, élabore le tableau d'une enfance victime de la guerre, mais dans un environnement qu'il a élaboré dans ses fictions précédentes: celui de Celama, de ses villes labyrinthiques, de ses habitants en déroute, luttant pour la survie.

Les noms communs employés d'une manière indéterminée et avec majuscules, tels que la Contienda, el Barrio, montrent la volonté de l'auteur de donner une valeur mythique à ce récit et, malgré un contexte qui rappelle la Guerre Civile espagnole, Luis Mateo Díez confie dans un entretien publié dans El Norte de Castilla, qu'il n'a pas voulu faire un roman sur la guerre civile. Pulgar, pour le romancier «Es un niño del desamparo, un niño de la orfandad, de la desgracia, que me gustaría que se pudiera ver un poco con el rostro de tantos otros niños actuales que están sufriendo en el mundo» (Mendoza 2007).

L'altruisme de Pulgar lui permet de montrer la permanence de la bonté, de la générosité malgré les horreurs de la guerre. Il présente, rappelant le conte tel que le définit Mircea Eliade, «l'initiation, c'est-à-dire le passage, par le truchement d'une mort et d'une résurrection symboliques, de la nescience et de l'immaturité à l'âge spirituel de l'adulte» (Eliade: 246). Le conte permet donc de donner une leçon de vie, de dire que "toute existence se constitue par une suite ininterrompue d'“épreuves", de "morts" et de "résurrections" " (Eliade: 248) comme souvent dans Luis Mateo Díez, malgré l'apparent défaitisme des personnages. 
Tout en se basant sur des emprunts à une tradition littéraire qui nous font adhérer à l'univers proposé, celui-ci se fonde sur une scripturalité propre à Luis Mateo Díez. Il s'agit bien d'une réappropriation d'un substrat littéraire existant pour créer une autre réalité fictionnelle.

\section{Bibliographie}

Barthes R., 1977, «Introduction à l'analyse structurale des récits», in Poétique du récit, Paris, Seuil, p. 7-57.

Belmont N. 1999, Poétique du conte, Essai sur le conte de tradition orale, Paris, Gallimard.

Bettelheim B., 1976, Psychanalyse des contes de fées, Paris, Robert Laffont.

Díez L. M., 2007, La gloria de los niños, Madrid, Alfaguara.

Díez L. M., 1991, Relato de Babia, Madrid, Espasa Calpe, Colección Austral nº 213.

Díez R. M., Díez Taboada P., 1998, La memoria de los cuentos, Un viaje por los cuentos populares del mundo, Madrid, Espasa Calpe, Colección Austral n ${ }^{\circ} 151$.

Eliade M., 1963, Aspects du mythe, Paris, Gallimard.

Larivaille P., 1974, «L’analyse (morpho)logique du récit», Poétiques, n 19, Paris, Seuil, p. 369-379.

Mendoza A., 2007, «Luis Mateo Díez reflexiona sobre la inocencia en su novela 'La gloria de los niños'», El Norte de Castilla, 23.10.07, http://www.elnortedecastilla. es/0071023/cultura/luis-mateo-diez-reflexiona-20071023.html

Mourey L., 1978, Introduction aux contes de Grimm et de Perrault: histoire, structure, mise en texte, Paris, Lettres modernes.

Propp V., 1970, Morphologie du conte, Paris, Seuil.

Saupé Y., 1997, Les Contes de Perrault et la mythologie, Rapprochements et influences, Paris-Seattle-Tübingen, Biblio 17.

Todorov T., 1981, «Les catégories du récit littéraire», in Communications, 8 , L'analyse structurale du récit, Paris, Seuil, (1966), p. 131-157.

Von Franz M-L., 1990, L'ombre et le mal dans les contes de fées, Paris, J. Renard: Éditions du dauphin, (1976).

Von Franz M-L., 2003, L'interprétation des contes de fées, Paris, J. Renard: Éditions Du dauphin, (1978). 